\title{
Confocal laser endomicroscopy for the in vivo detection of intraepithelial neoplasia in Peutz- Jeghers polyps
}
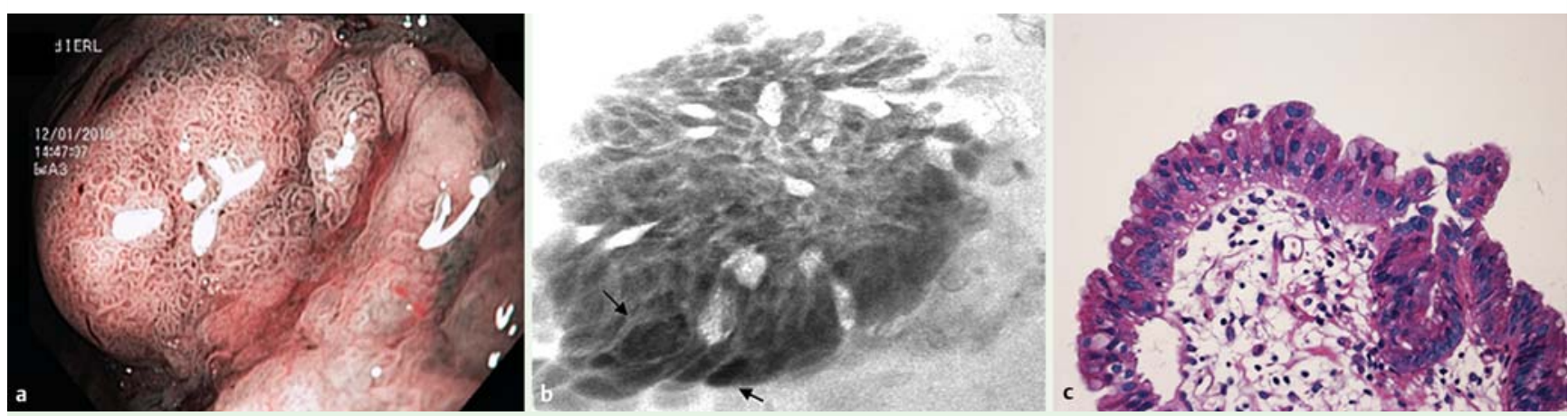

Fig. 1 a Esophagogastroduodenoscopy with narrow-band imaging exposed a large polyp in the gastric fundus. b Confocal laser endomicroscopy showed large and irregular cells with increased optical density and fluorescein leakage. c Histopathological analysis revealed focal high-grade intraepithelial neoplasia.
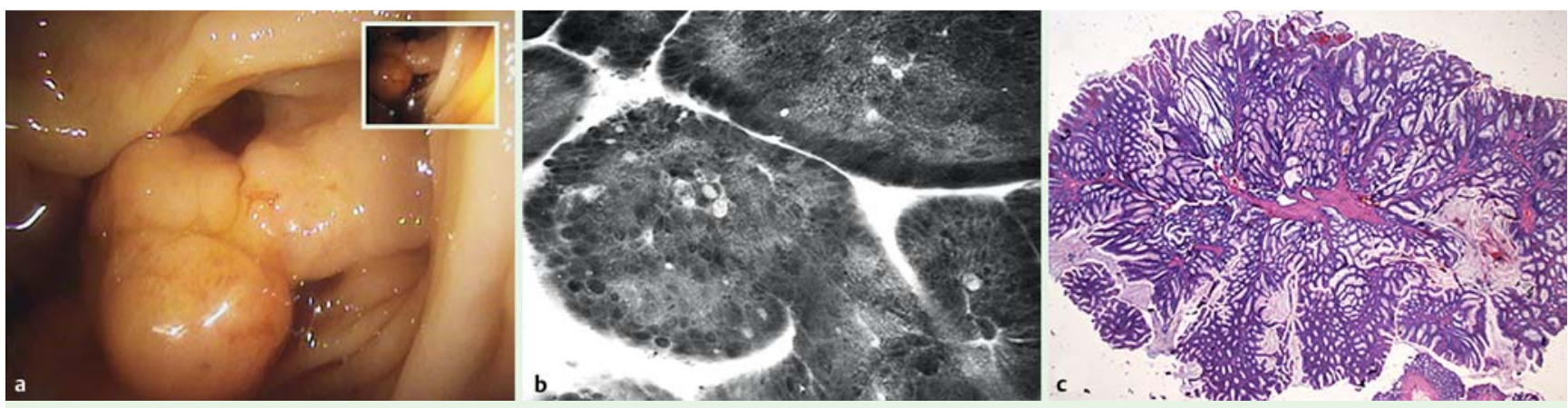

Fig. 2 a Large, pedunculated polyp in the proximal sigmoid. b As well as regenerative changes, confocal laser endomicroscopy revealed disorganization of glands, occurrence of different cell types, and the presence of muscle fibers. $\mathbf{c}$ Histopathological examination showed an overall villous architecture and markedly interdigitating smooth muscle bundles of varying thickness, which arose from the muscularis mucosae.

Peutz-Jeghers syndrome (PJS) is a rare, autosomal dominant condition that is characterized by hamartomatous polyps in the gastrointestinal tract and mucocutaneous melanin pigmentation $[1,2]$. Confocal laser endomicroscopy (CLE) is rapidly emerging as a valuable tool for gastrointestinal endoscopic imaging, enabling the endoscopist to obtain an "optical biopsy" of the gastrointestinal mucosa during the endoscopic procedure [3-5].

Here, we present the case of a patient with genetically confirmed PJS who was referred for surveillance endoscopy. Esophagogastroduodenoscopy revealed a large, polypoid mass of about $20 \mathrm{~mm}$ in diameter located in the gastric fundus $(\bullet$ Fig. 1a). Pit pattern analysis using narrow-band imaging (Olympus, Tokyo, Japan) revealed large roundish and tubular pits (Kudo $3 \mathrm{~L}$ ). After administration of $3 \mathrm{~mL}$ intravenous fluorescein 10\% (Alcon Laboratories, Texas, USA), CLE (EG-3870
CIK, Pentax, Tokyo, Japan) was performed. Endoscopic histology revealed large, irregular cells with increased optical density and fluorescein leakage ( $\boldsymbol{\bullet}$ Fig. $\mathbf{1 b}$ ). According to these changes the diagnosis of high-grade intraepithelial neoplasia was made. Corresponding histopathological analysis confirmed the presence of low-grade intraepithelial neoplasia and a focal high-grade intraepithelial neoplasia as well ( $\bullet$ Fig. 1 c).

Colonoscopy (Pentax EC-3870 CIFK, Tokyo, Japan) showed seven polyps ranging from $2 \mathrm{~mm}$ to $15 \mathrm{~mm}$ in diameter ( 0 Fig. 2a). CLE of the polyps showed disorganization of glands, occurrence of different cell types, the presence of muscle fibers, some regenerative changes but no atypia ( $\bullet$ Fig. 2b). Histopathological examination confirmed the findings showing an overall villous architecture and markedly interdigitating smooth muscle bundles of varying thickness, which arose from the muscularis mucosae and extended into the head of the polyp ( $\bullet$ Fig. $\mathbf{2 c}$ ).

This is the first report of CLE in diagnosing polyps in PJS. Our case highlights the utility of CLE for the in vivo analysis of intestinal polyps. Thus, CLE may significantly improve the clinicohistopathologic evaluation and may prevent diagnostic and therapeutic delays.

\section{Competing interests: None}

Endoscopy_UCTN_Code_CCL_1AD_2AC

\section{H. Neumann ${ }^{1}$, M. Vieth ${ }^{2}$, M. Raithel',} J. Mudter ${ }^{1}$, R. Kiesslich ${ }^{3}$, M. F. Neurath ${ }^{1}$ Department of Medicine I, University of Erlangen-Nuremberg, Germany 2 Institute of Pathology, Klinikum Bayreuth, Germany

3 I Medical Clinic, Johannes Gutenberg University of Mainz, Germany 


\section{References}

1 Giardiello FM, Trimbath JD. Peutz-Jeghers syndrome and management recommendations. Clin Gastroenterol Hepatol 2006; 4: $408-415$

2 Harbaum L, Geigl JB, Volkholz H et al. Sporadic gastric Peutz-Jeghers polyp with intraepithelial neoplasia. APMIS 2009; 117: 941-943

3 Kiesslich R, Goetz M, Neurath MF. Virtual histology. Best Pract Res Clin Gastroenterol 2008; 22: 883-897
4 Buchner AM, Shahid MW, Heckman MG et al. Comparison of probe-based confocal laser endomicroscopy with virtual chromoendoscopy for classification of colon polyps. Gastroenterology 2010; 138: 834-842

5 Li WB, Zuo XL, Zuo F et al. Characterization and identification of gastric hyperplastic polyps and adenomas by confocal laser endomicroscopy. Surg Endosc 2010; 24: 517 524

\section{Bibliography}

DOI $10.1055 / \mathrm{s}-0029-1244049$

Endoscopy 2010; 42: E139-E140

(c) Georg Thieme Verlag KG Stuttgart · New York . ISSN 0013-726X

\section{Corresponding author}

H. Neumann, MD, PhD

Department of Medicine I

University of Erlangen-Nuremberg

Ulmenweg 18

91054 Erlangen

Germany

Fax: +49-9131-8535209

helmut.neumann@uk-erlangen.de 